\title{
Modeling of Nonlinear Autoregressive Neural Network for Multi-Step Ahead Air Quality Prediction
}

\author{
Mirza Pasic, Izet Bijelonja, Edin Kadric, Hadis Bajric \\ Mechanical Engineering Faculty Sarajevo, University of Sarajevo, Vilsonovo setaliste 9, \\ Sarajevo, Bosnia and Herzegovina
}

\begin{abstract}
In this paper five neural network models were developed using NARX-SP neural network type in order to predict air pollutants concentrations $\left(\mathrm{SO}_{2}\right.$, $\mathrm{PM}_{10}, \mathrm{NO}_{2}, \mathrm{O}_{3}$ and $\mathrm{CO}$ ) for the 72nd hour ahead for Sarajevo. Hourly values of air pollutants concentrations and meteorological parameters (air temperature, pressure and humidity, wind speed and direction) for Sarajevo were used. Optimal model was selected based on the values of $R^{2}, M S E$ and the complexity of models. Optimal neural network model can predict air pollutants concentrations for the $72 \mathrm{nd}$ hour ahead with high accuracy, as well as for all hours up to 72nd hour.
\end{abstract}

Keywords - NARX-SP, neural network, air pollutant concentration, meteorological parameters

\section{Introduction}

The increasing possibility to collect large amounts of data about particular phenomena, as well as the development of computers and the power of its processors, have enabled usage of scientific methods for analysing and establishing relationships between data, which help better understanding certain phenomena and make adequate decisions. One of the scientific methods that has proven very effective in solving such tasks are neural networks.

DOI: $10.18421 /$ TEM93-03

https://doi.org/10.18421/TEM93-03

Corresponding author: Mirza Pasic, Mechanical Engineering Faculty Sarajevo,

University of Sarajevo, Bosnia and Herzegovina

Email: mirza.pasic@mef.unsa.ba

Received: 07 May 2020.

Revised: 24 June 2020.

Accepted: 01 July 2020.

Published: 28 August 2020.

(cc) BY-NC-ND_C 2020 Mirza Pasic, Izet Bijelonja, Edin Kadric \& Hadis Bajric; published by UIKTEN. This work is licensed under the Creative Commons AttributionNonCommercial-NoDerivs 4.0 License.

The article is published with Open Access at www.temjournal.com
Neural networks are based on the work of neurons in humans and are suitable for use in the presence of large amounts of data. They can predict, with great accuracy, certain phenomena based on data related to that phenomena. The development of neural networks has proven to be extremely useful for cases where many other methods have proven insufficient.

Air quality is one of the phenomena where a large amount of data is collected. The prediction of air quality refers to the prediction of the concentration of air pollutants. Poor air quality is associated with a wide range of diseases, from acute to chronic, and from less dangerous to those very life-threatening. This problem is especially present in Sarajevo, where the air quality index is often in the danger zone. Due to the high impact of polluted air on human health, this issue is attracting a lot of public attention.

According to the U.S. Environmental Protection Agency (U.S. EPA), 187 pollutants have been identified that can cause health problems in humans. U.S. EPA has identified 6 most dangerous pollutants for human health and those are particulate matter $\left(P M_{10}, P M_{2,5}\right)$, ozone at ground level $\left(O_{3}\right)$, carbon monoxide $(\mathrm{CO})$, sulfur dioxide $\left(\mathrm{SO}_{2}\right)$, lead $(\mathrm{Pb})$ and nitrogen dioxide $\left(\mathrm{NO}_{2}\right)$ [1].

From the literature reviewed, it can be concluded that different types of neural networks for different geographical locations, as well as different combinations of inputs and outputs were used to predict the concentrations of different pollutants in the air. Adams and Kanaraoglou [2] conducted research for the city of Hamilton, Ontario, Canada using ANN. Alimissis et al. [3] analyzed multiple urban sites in Greece. Zhang and Li [4] analyzed air pollution in Xi'an and Shanghai in China.

Kolehmainen, Martikainen and Ruuskanen [5] used feedforward neural network (FFNN) with backpropagation algorithm to predict the concentration of $\mathrm{NO}_{2}$ in Stockholm. Cai, Yin and Xie [6] used FFNN to predict hourly values of $\mathrm{CO}, \mathrm{NO}_{2}$, $P M_{10}$, and $O_{3}$. This type of network was also used by Viotti, Liuiti and Di Genova [7] to predict urban pollution. 
A review of the literature revealed that one of the most powerful types of neural networks is the nonlinear autoregressive exogenous network (NARX). The NARX neural network is a type of neural network that is adequate in working with data where the values of the predicted outputs depend on their values from the previous period, as well as the values of other inputs from that period [8].

The data required for model development were taken from the Federal Meteorological Institute of Bosnia and Herzegovina and represent hourly values of meteorological parameters (air temperature, pressure and humidity, wind speed and direction), as well as pollutant concentrations $\left(\mathrm{SO}_{2}, \mathrm{PM}_{10}, \mathrm{NO}_{2}\right.$, $\mathrm{O}_{3}$ and $\mathrm{CO}$ ) for the period from the beginning of 2016 to the end of 2018.

For the development of neural network models, a code in Python was created using the Keras library.

Neural network models for the prediction of air pollutant concentrations for the 72nd hour ahead from the current hour with a different combination of inputs and outputs for Sarajevo were developed.

For the prediction of concentration of $S_{2}, P M_{10}$, $\mathrm{NO}_{2}, \mathrm{O}_{3}$ and $\mathrm{CO}$, concentration of these pollutants from the previous period, as well as the values of meteorological parameters, month, day and time in that day from the same previous period were used.

Different combinations of inputs and outputs were achieved by different number of hours from the previous period from which data were used to develop neural network models.

Depending on the number of hours from the previous period used in the development of neural networks, 5 different models were developed.

An optimal model was selected based on the values of the coefficient of determination $R^{2}$ and the mean squared error $M S E$ for the test data set and the complexity of the model. For the optimal neural network model, the relations between observed and predicted air pollutants concentration values for all data, for each pollutant were graphically presented.

With the optimal model selected, the prediction of the air pollutants concentration for each hour in the next 72 hours can be made relative to the current hour by correctly selecting the referent hour against which the prediction is made.

\section{Theoretical Background}

In the modern world, in many areas of life, a large amount of data is being collected, and there is a need for new methods of data processing, called machine learning. Neural networks have been shown to be one of the most effective methods of machine learning for prediction of certain phenomena.

Neural networks operate on the principle of biological neuron operation [9], [10]. Using the principle of work of biological neurons, McCulloh and Pitts created a mathematical model of neurons (MCP).

This simple model of MCP neurons consists of a set of input data $x_{i}$, set of weights $w_{i}$ and activation function. The model works by multiplying the value of each input $x_{i}$ by the corresponding weight $w_{i}$, and then these products are summed up in equation (2.1) [9], [10]:

$$
h=\sum_{i=1}^{L} w_{i} \cdot x_{i}
$$

where:

$i$ - the order number of the input value $(i=1, \ldots, L)$.

The resulting sum $h$ enters the activation function, which has its own sensitivity threshold. The MCP neuron has a binary character, because the activation function is binary, so as an output value it can give is only 0 or 1, as shown in equation (2.2) [9], [10]:

$$
y=g(h)=\left\{\begin{array}{l}
1, \text { if } h \geq \theta \\
0, \text { if } h \leq \theta
\end{array}\right.
$$

Based on the principles of the MCP in 1958, scientist Rosenblatt invented perceptron, the first modern neural network. Perceptron is a set of multiple neurons with multiple weights and multiple outputs. The perceptron consists of an input and output layer and its structure is shown in Figure 1. [9], [10].



Figure 1. Perceptron (adapted from [9])

The importance of Rosenblatt's perceptron is the ability to learn. Learning implies that weights change until the best output results are achieved. This form of perceptron can only solve linear problems, so as the problems are much more complex in reality, more weights have to be introduced. In 1986, Rumelhart, McClelland and Hinton proposed the creation of a multi-layer perceptron (MLP), which has one or more hidden layers between the input and output layers. The correction of weights to their 
optimal value, in order to achieve the best performance of the neural network, is achieved using the backpropagation algorithm [9].

NARX neural networks have been shown to be very useful tools for modelling nonlinear systems as it is the case in this paper. The NARX neural network actually has the structure of a recurrent neural network, and it is used to model nonlinear dynamic systems as well as to predict time series [8], [11], [12].

There are two types of NARX neural networks, Serial-Parallel (NARX-SP) and Parallel (NARX-P) neural network. In this paper, the NARX-SP type of neural network was used. As inputs the previous output values and exogenous inputs from the same previous period were used. One area where the NARX-SP neural network can be successfully used is to predict the concentration of air pollutants [13], [14], [15].

Air pollution is the second largest environmental problem in the world after climate change. The importance of this problem has been recognized in the European Union (EU), and in organizations such as the United Nations (UN) and the World Health Organization (WHO). In order to reduce air pollution in the EU countries, various measures have been taken to improve air quality. These measures have resulted in significant improvements in air quality, although pollution is still a major problem. In some areas, such as urban areas, there has even been an increase in air pollution. Air pollution over permitted values increases mortality, shortens life expectancy, causes many diseases, increases medical costs, affects the economy and climate change. Pollutants in excessive concentration damage buildings and cultural monuments, and negatively affect water, soil, vegetation and wildlife [16].

The problem of air pollution is also present in Sarajevo. The most common air pollutants in Sarajevo are $\mathrm{SO}_{2}, \mathrm{PM}_{10}, \mathrm{NO}_{2}, \mathrm{O}_{3}$ and $\mathrm{CO}$. In order to protect human health, it is very important to know the concentration of air pollutants in the coming period, so that citizens can plan their activities accordingly. This can be achieved by accurately predicting the concentration of air pollutants for the coming period.

\section{Methodology}

The NARX-SP neural network type was used to develop the neural network model, and data were obtained from the Federal Meteorological Institute of $\mathrm{BiH}$. The data consists of hourly values of meteorological parameters, air temperature $(\boldsymbol{T})$, pressure $(\boldsymbol{p})$ and humidity $(\boldsymbol{H})$, wind speed $(\boldsymbol{v})$ and wind direction $(\boldsymbol{w d})$ and concentration of air pollutants $\boldsymbol{S O}_{2}, \boldsymbol{P M}_{10}, \mathrm{NO}_{2}, \boldsymbol{O}_{3}$ and $\mathrm{CO}$, for the period from the beginning of 2016 to the end of 2018. Data were processed, so the database contains 26304 data for each meteorological parameter and for each air pollutant.

In order to gain a better understanding of the data used in this work for the development of neural network models, Table 1 presents descriptive statistics of these data (mean, median, mode, standard deviation, minimum and maximum values, and units).

Table 1. Descriptive statistics of used data

\begin{tabular}{|c|c|c|c|c|c|c|c|c|c|c|}
\hline & $H$ & $p$ & $T$ & $v$ & $w d$ & $\mathrm{SO}_{2}$ & $P M_{10}$ & $\mathrm{NO}_{2}$ & $\mathrm{O}_{3}$ & $\mathrm{CO}$ \\
\hline Mean & 71,159 & 944,222 & 11,064 & 1,870 & 18,635 & 15,286 & 41,297 & 20,914 & 58,427 & 0,305 \\
\hline Median & 75,000 & 944,300 & 11,400 & 1,600 & 15,839 & 6,900 & 26,100 & 14,350 & 53,900 & 0,170 \\
\hline Mode & 88,000 & 945,000 & 13,200 & 0,900 & 11,000 & 6,965 & 24,149 & 11,098 & 69,958 & 0,040 \\
\hline $\begin{array}{l}\text { Standard } \\
\text { deviation }\end{array}$ & 17,797 & 6,957 & 9,058 & 1,134 & 8,951 & 27,061 & 51,186 & 21,424 & 38,491 & 0,431 \\
\hline Minimum & 7,000 & 841,800 & 22,200 & 0,000 & 2,000 & 0,000 & 0,000 & 0,000 & 0,000 & 0,000 \\
\hline Maximum & 100,000 & 993,400 & 44,000 & 15,500 & 36,000 & 872,300 & 756,100 & 271,600 & 434,600 & 5,760 \\
\hline Unit & $\%$ & $\mathrm{hPa}$ & ${ }^{\circ} \mathrm{C}$ & $\mathrm{m} / \mathrm{s}$ & & $\mu \mathrm{g} / \mathrm{m}^{3}$ & $\mu \mathrm{g} / \mathrm{m}^{3}$ & $\mu \mathrm{g} / \mathrm{m}^{3}$ & $\mu \mathrm{g} / \mathrm{m}^{3}$ & $\mu \mathrm{g} / \mathrm{m}^{3}$ \\
\hline
\end{tabular}

The data used to develop neural network models have been previously adequately sorted, normalized and filtered. The values of the concentration of air pollutants and meteorological parameters other than wind direction (wd) are normalized to the interval $[0,1]$, by the algorithm created in Python shown in Figure 2. 


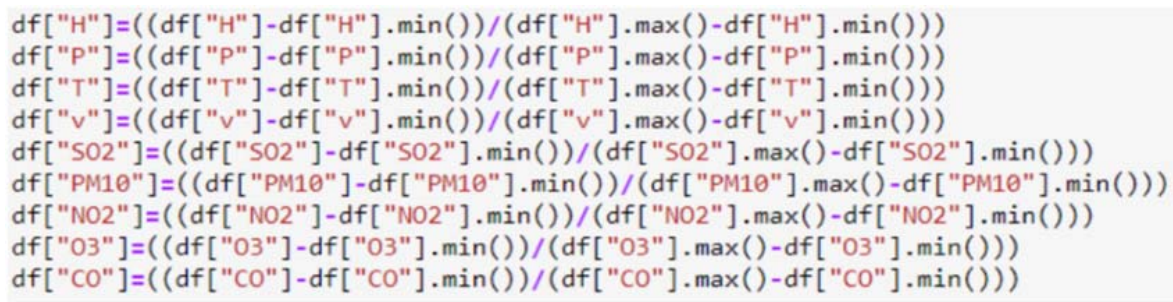

Figure 2. Code in Python for normalizing data

The values referring to the month of the year, day of the week, time of a day and wind direction were normalized to the interval $[-1,1]$ using the sin and cos functions. This means that the sin and $\cos$ components of the month, day, time of a day and wind direction were used in further work.

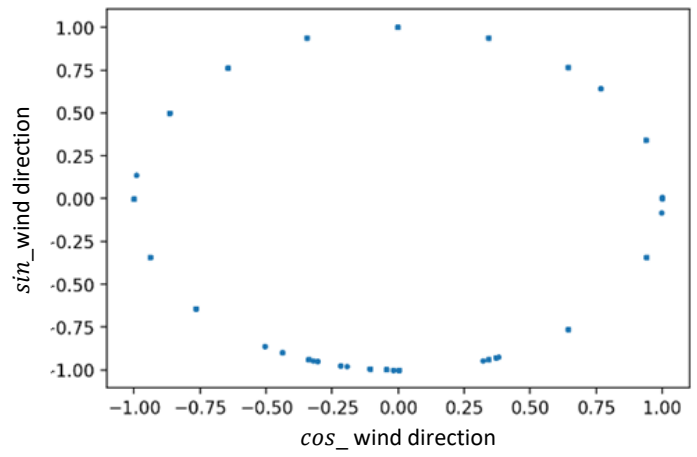

a)

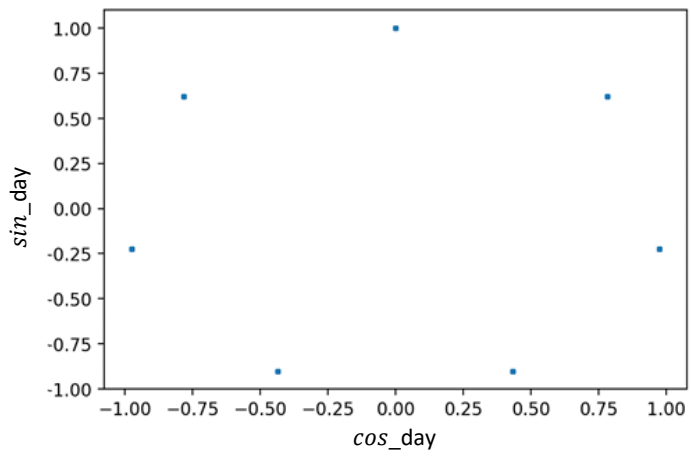

c)
These values are normalized in this way because of their cyclic repetition, where the last data in the sequence has an effect on the first data, as shown in Figure 3.

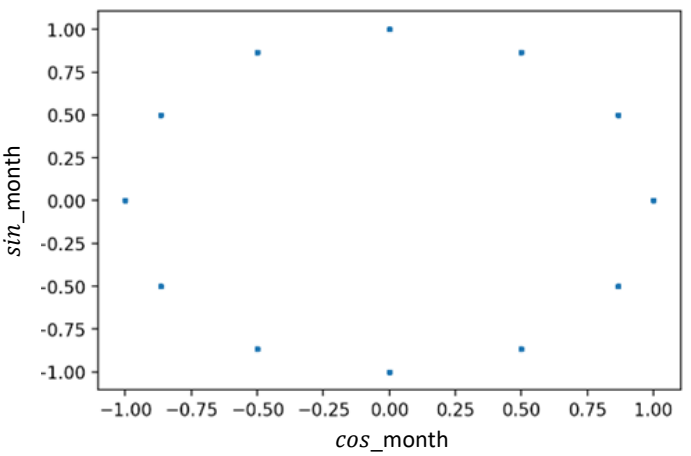

b)

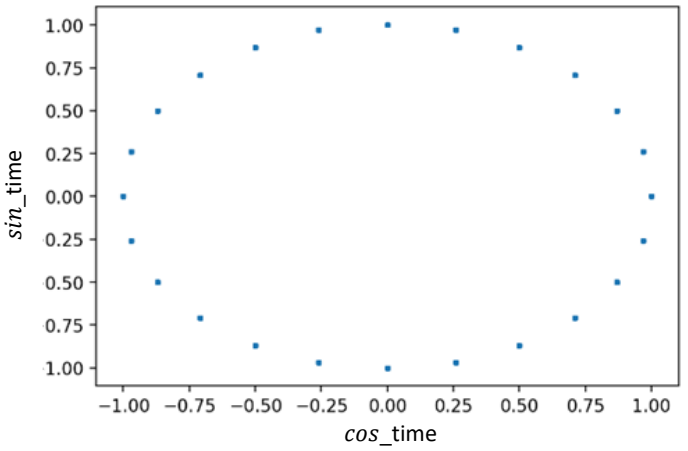

d)

Figure 3. Cyclic repetition for: a) wind direction, b) month, c) day and d) time

The sigmoid function was used as an activation function in the layers of all developed neural network models. The batch size of 10, 500 epochs with early stopping and the Adam optimizer were also used.

The number of neurons in the input layer depends on the selected number of previous hours from which the prediction data were used. In each developed model, meteorological parameters, month of the year, day of the month and time in that day for the hour for which the prediction is made (12 inputs) and meteorological parameters, concentration of air pollutants, month, day and time in that day from the referent hour were used as inputs (17 inputs), and from the referent hour and the selected number of previous hours.

The data is split into three sets, training, validation and test data set. For the developed models the values of the coefficient of determination $R^{2}$ and the mean squared error $M S E$ were calculated. Optimal neural network model was selected based on values of $R^{2}$, $M S E$ and model complexity. Relations between the predicted and observed values of concentration of air pollutants were graphically presented, and conclusions were made about the prediction accuracy of the selected optimal model.

The inputs to the neural network were the values of meteorological parameters, concentration of air pollutants, month in the year, day of the week and time of a day from the referent hour and the referent hour, and previous 23, 35, 47 and 71 hours and values of meteorological parameters, month, day and time for the hour for which the prediction is made.

Structures of models in terms of the number of neurons in the input, hidden and output layers is shown in Table 2. 
Table 2. Structures of neural network models for the 72nd hour ahead

\begin{tabular}{|c|c|c|}
\hline Model & Number of previous hours & Structure \\
\hline 1 & Referent hour & $29-59-5$ \\
\hline 2 & Referent hour +23 previous hours & $432-865-5$ \\
\hline 3 & Referent hour +35 previous hours & $636-1273-5$ \\
\hline 4 & Referent hour +47 previous hours & $864-1729-5$ \\
\hline 5 & Referent hour +71 previous hours & $1296-2593-5$ \\
\hline
\end{tabular}

\section{Results and Discussion}

Model analysis was performed based on the values of $R^{2}$ and MSE. The $R^{2}$ and MSE values of all 5

models for all data sets and for all pollutants are shown from Tables 3. to 10.

Table 3. $R^{2}$ values [\%] for training data set

\begin{tabular}{|c|c|c|c|c|c|c|}
\hline Model & $R^{2}$ train & $R^{2}$ train $\mathrm{SO}_{2}$ & $R^{2} \operatorname{train} P M_{10}$ & $R^{2}$ train $N O_{2}$ & $R^{2}$ train $O_{3}$ & $R^{2}$ train $C O$ \\
\hline 1 & 62,805 & 43,544 & 59,247 & 74,858 & 62,700 & 73,674 \\
\hline 2 & 96,250 & 93,877 & 96,342 & 96,971 & 96,303 & 97,757 \\
\hline 3 & 97,437 & 95,923 & 97,241 & 97,645 & 97,969 & 98,408 \\
\hline 4 & 96,921 & 94,690 & 96,891 & 97,644 & 97,184 & 98,197 \\
\hline 5 & 96,825 & 94,143 & 96,908 & 97,662 & 97,852 & 97,829 \\
\hline
\end{tabular}

Table 4. MSE values for training data set

\begin{tabular}{|c|c|c|c|c|c|c|}
\hline Model & $M S E$ train & $M S E$ train $S_{2}$ & $M S E$ train $P M_{10}$ & $M S E$ train $N_{2}$ & $M S E$ train $O_{3}$ & $M S E$ train $C O$ \\
\hline 1 & 0,002184728 & 0,001533912 & 0,002136510 & 0,002255586 & 0,003401855 & 0,001595778 \\
\hline 2 & 0,000223554 & 0,000167367 & 0,000198091 & 0,000266049 & 0,000347346 & 0,000138917 \\
\hline 3 & 0,000151678 & 0,000115821 & 0,000151494 & 0,000204890 & 0,000189124 & 0,000097059 \\
\hline 4 & 0,000179314 & 0,000144191 & 0,000169546 & 0,000211200 & 0,000262457 & 0,000109173 \\
\hline 5 & 0,000171987 & 0,000161406 & 0,000162326 & 0,000191556 & 0,000224409 & 0,000120235 \\
\hline
\end{tabular}

Table 5. $R^{2}$ values [\%] for validation data set

\begin{tabular}{|c|c|c|c|c|c|c|}
\hline Model & $R^{2}$ _val & $R^{2}$ val $S_{2}$ & $R^{2}$ val $P M_{10}$ & $R^{2}$ val $\mathrm{NO}_{2}$ & $R^{2}$ val $O_{3}$ & $R^{2}$ _val_CO \\
\hline 1 & 60,065 & 42,333 & 56,511 & 71,880 & 61,096 & 68,504 \\
\hline 2 & 91,693 & 84,781 & 93,759 & 93,686 & 91,018 & 95,220 \\
\hline 3 & 93,773 & 89,498 & 95,031 & 94,583 & 93,271 & 96,480 \\
\hline 4 & 92,707 & 86,810 & 93,624 & 93,561 & 93,242 & 96,299 \\
\hline 5 & 92,522 & 86,558 & 94,238 & 93,133 & 93,725 & 94,956 \\
\hline
\end{tabular}

Table 6. MSE values for validation data set

\begin{tabular}{|c|c|c|c|c|c|c|}
\hline Model & $M S E \_$val & $M S E \_$val $S O_{2}$ & $M S E \_$val_PM10 & $M S E \_$val_NO & $M S E$ val $O_{3}$ & $M S E \_$val_CO \\
\hline 1 & 0,002428519 & 0,001636032 & 0,002447507 & 0,0021517785 & 0,003629123 & 0,001912149 \\
\hline 2 & 0,000474193 & 0,000375878 & 0,000345137 & 0,000556281 & 0,000805195 & 0,000288473 \\
\hline 3 & 0,000368757 & 0,000251832 & 0,000266125 & 0,000484383 & 0,000632428 & 0,000209017 \\
\hline 4 & 0,000411553 & 0,000337954 & 0,000338350 & 0,000537356 & 0,000617833 & 0,000226270 \\
\hline 5 & 0,000447001 & 0,000360522 & 0,000331294 & 0,000636636 & 0,000578712 & 0,000327840 \\
\hline
\end{tabular}

Table 7. $R^{2}$ values [\%] for test data set

\begin{tabular}{|c|c|c|c|c|c|c|}
\hline Model & $R^{2}$ test & $R^{2}$ test $\mathrm{SO}_{2}$ & $R^{2}$ test $P M_{10}$ & $R^{2}$ test $\mathrm{NO}_{2}$ & $R^{2}$ test $\mathrm{O}_{3}$ & $R^{2}$ test $C O$ \\
\hline 1 & 60,852 & 47,234 & 56,017 & 71,235 & 58,962 & 70,813 \\
\hline 2 & 92,204 & 88,017 & 93,241 & 93,684 & 90,884 & 95,194 \\
\hline 3 & 93,403 & 89,517 & 94,364 & 93,702 & 92,995 & 96,439 \\
\hline 4 & 92,862 & 87,344 & 93,886 & 93,459 & 93,437 & 96,183 \\
\hline 5 & 93,025 & 87,101 & 94,663 & 93,978 & 93,338 & 96,046 \\
\hline
\end{tabular}


Table 8. MSE values for test data set

\begin{tabular}{|c|c|c|c|c|c|c|}
\hline \multirow{2}{*}{ Model } & $M S E_{-}$test & $\begin{array}{c}M S E_{-} \text {test } \\
S O_{2}\end{array}$ & $\begin{array}{c}M S E_{-} \text {test } \\
\overline{P M}_{10}\end{array}$ & $\begin{array}{c}M S E_{-} \text {test } \\
N O_{2}\end{array}$ & $\begin{array}{c}M S E_{-} \text {test } \\
\bar{O}_{3}\end{array}$ & $\begin{array}{c}M S E_{-} \text {test } \\
C O\end{array}$ \\
\hline 1 & 0,002528202 & 0,001538079 & 0,002547798 & 0,002580728 & 0,004014633 & 0,001959770 \\
\hline 2 & 0,000489889 & 0,000352507 & 0,000361692 & 0,000588074 & 0,000847853 & 0,000299317 \\
\hline 3 & 0,000402715 & 0,000263937 & 0,000298198 & 0,000586891 & 0,000636526 & 0,000228021 \\
\hline 4 & 0,000411997 & 0,000335452 & 0,000332844 & 0,000550051 & 0000610238 & 0,000231399 \\
\hline 5 & 0,000406506 & 0,000296979 & 0,000297544 & 0,000549379 & 0,000619560 & 0,000269065 \\
\hline
\end{tabular}

Table 9. $R^{2}$ values [\%] for all data

\begin{tabular}{|c|c|c|c|c|c|c|}
\hline Model & $R^{2}$ all & $R^{2}$ all $S_{2}$ & $R^{2}$ all $P M_{10}$ & $R^{2}$ all $N_{2} O_{2}$ & $R^{2}$ all $O_{3}$ & $R^{2}$ all $C O$ \\
\hline 1 & 61,849 & 44,079 & 57,991 & 73,538 & 61,591 & 72,045 \\
\hline 2 & 94,534 & 90,939 & 95,194 & 95,621 & 94,180 & 96,735 \\
\hline 3 & 95,923 & 93,552 & 96,234 & 96,186 & 96,025 & 97,615 \\
\hline 4 & 95,291 & 91,706 & 95,642 & 96,048 & 95,649 & 97,408 \\
\hline 5 & 95,165 & 91,347 & 95,866 & 95,882 & 95,944 & 96,786 \\
\hline
\end{tabular}

Table 10. MSE values for all data

\begin{tabular}{|c|c|c|c|c|c|c|}
\hline \multirow{2}{*}{ Model } & $M S E_{-}$test & $\begin{array}{c}M S E_{-} \text {test } \\
S \bar{O}_{2}\end{array}$ & $\begin{array}{c}M S E_{-} \text {test } \\
P M_{10}\end{array}$ & $\begin{array}{c}M S E_{-} \text {test } \\
N O_{2}\end{array}$ & $\begin{array}{c}M S E_{-} \text {test } \\
\bar{O}_{3}\end{array}$ & $\begin{array}{c}M S E_{-} \text {test } \\
C O\end{array}$ \\
\hline 1 & 0,002302290 & 0,001555052 & 0,002281075 & 0,002373119 & 0,003570307 & 0,001731897 \\
\hline 2 & 0,000327847 & 0,000246744 & 0,000260767 & 0,000389578 & 0,000540696 & 0,000201448 \\
\hline 3 & 0,000246215 & 0,000173210 & 0,000204258 & 0,000338426 & 0,000369066 & 0,000146116 \\
\hline 4 & 0,000272778 & 0,000221591 & 0,000236305 & 0,000344896 & 0,000403808 & 0,000157288 \\
\hline 5 & 0,000275242 & 0,000229300 & 0,000223987 & 0,000354306 & 0,000376093 & 0,000192523 \\
\hline
\end{tabular}

It can be seen from Tables 3 to 10 that for all developed neural network models, the values of $R^{2}$ and $M S E$ for both training and validation data sets show approximately the same values as for the test set. It can be concluded that the developed neural network models performed the learning process correctly.

Figures 4 and 5 graphically represent the values of $R^{2}$ and $M S E$ by models for the test data set. The values of $R^{2}$ for the test data set in Table 7 differ very little from each other for models 2, 3, 4 and 5, as can be seen from Figure 4. Only model 1, where the prediction is based on the data from the referent hour and the hour for which the prediction is being made, has significantly lower the value of $R^{2}$ than the other models. Sharp increase in the value of $R^{2}$, from $60,852 \%$ to $92,204 \%$ can be observed between models 1 and 2, because model 2 used more data from the previous period as it is shown in Table 2 . It shows the fact that in order to predict the concentration of air pollutants for the 72nd hour, it is necessary to provide more data from the previous period. By comparing models 2, 3, 4 and 5, it can be seen that model 3 has the highest value of $R^{2}=$ $93,403 \%$.

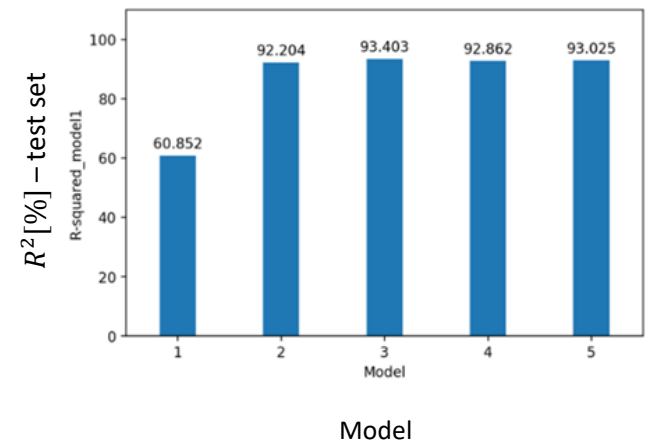

Figure 4. $R^{2}$ values [\%] for test set by models

From Figure 5, and by analyzing the values in Table 8 for the test data set, a sharp decrease in the $M S E$ value between model 1 and model 2 can be observed, from $M S E=0,002528202$ to $M S E=$ 0,000489889. For models 2, 3, 4 and 5, the $M S E$ values are approximately the same, with model 3 having the lowest value of $M S E=0,000402715$.

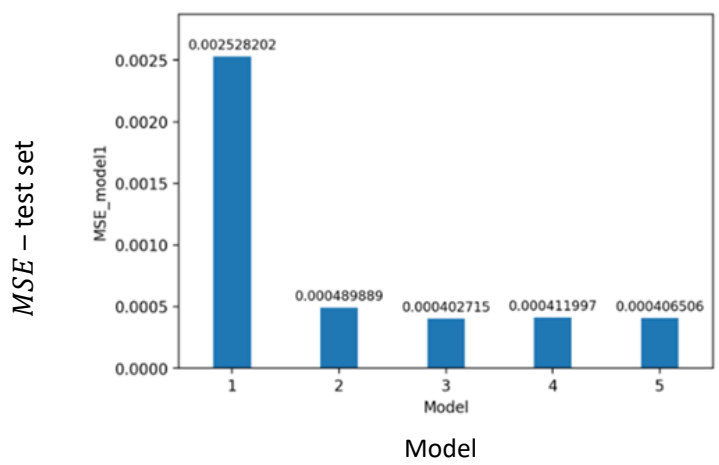

Figure 5. MSE values for test set by models 
Model 3 has the highest $R^{2}$ value and the lowest $M S E$ value, so it was selected as the optimal model for prediction the concentration of air pollutants for the $72 \mathrm{nd}$ hour ahead. Based on the values of $R^{2}=$ $93,403 \%$ and $M S E=0,000402715$ for model 3 for the test data set, it can be concluded that model 3 has very high values of $R^{2}$ and low values of $M S E$ performance. By analyzing the values of $M S E$ for training and validation data set during the learning process, it was examined whether the network was overtrained for the selected model 3 , as shown in Figure 6.

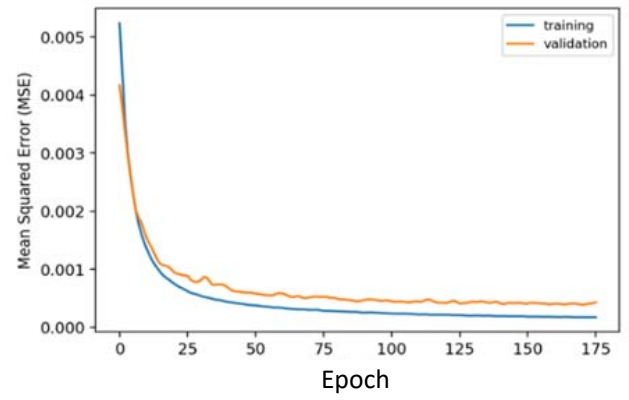

Figure 6. Change of MSE values by epochs for training and validation set (model 3)

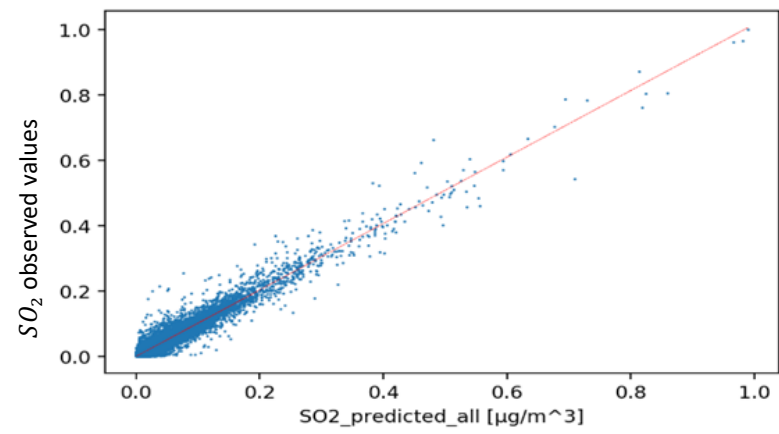

$\mathrm{SO}_{2}$ predicted values (normalised) - all data

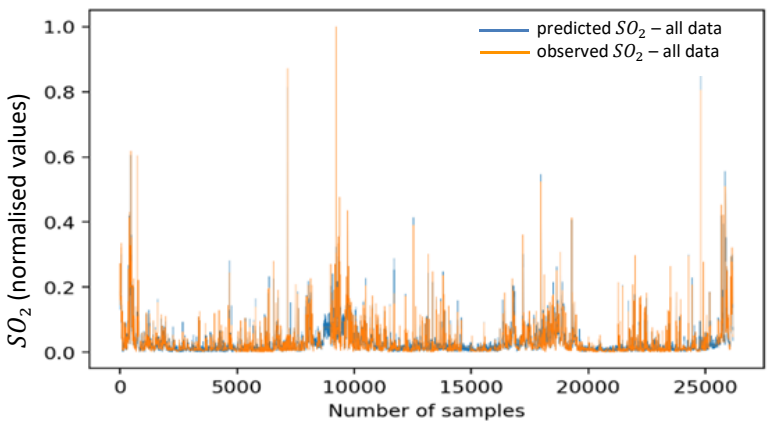

Sample

Figure 7. Relation between observed and predicted values $\mathrm{SO}_{2}$ for all data
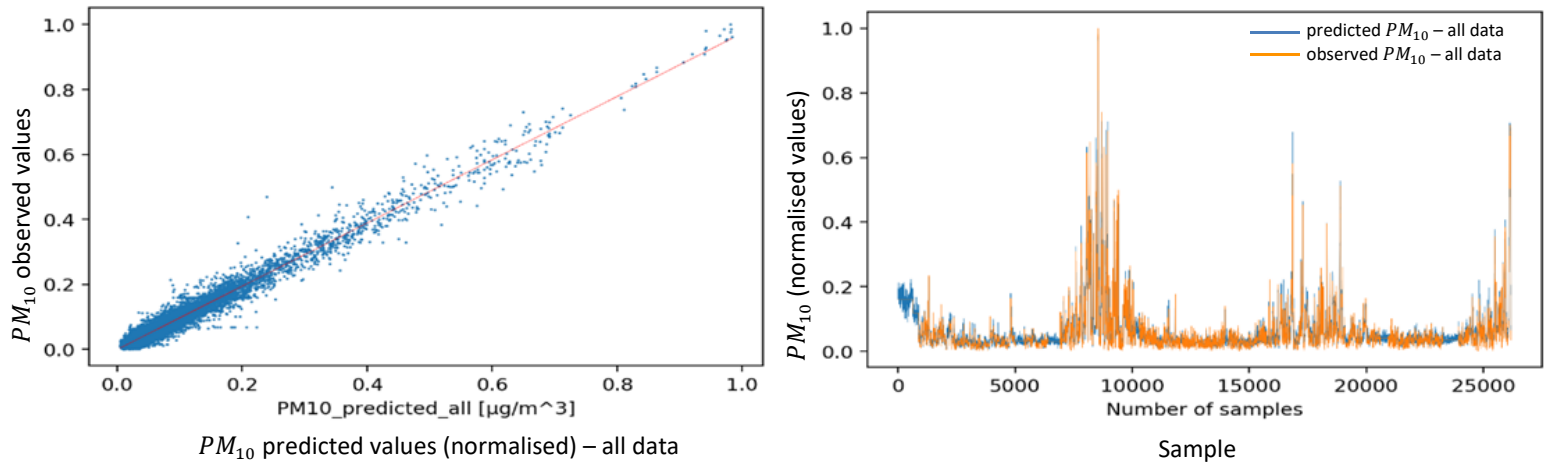

Figure 8. Relation between observed and predicted values $P M_{10}$ for all data
Figure 6 shows that the learning process stopped in the 175th epoch. Based on the movement of the $M S E$ curves for the validation and training set, it can be concluded that the learning process was done correctly, ie. that the network is not overtrained. For the selected optimal model 3 , as the best performing model, the relations of predicted and observed values for all data for each pollutant individually were graphically presented in Figures 7 to 11 . 


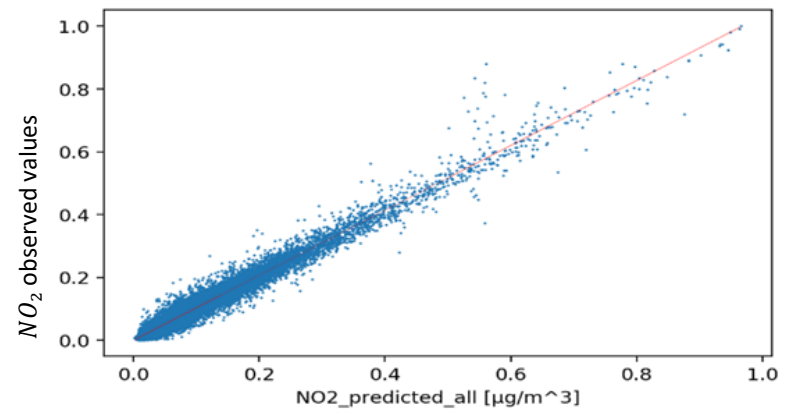

$\mathrm{NO}_{2}$ predicted values (normalised) - all data

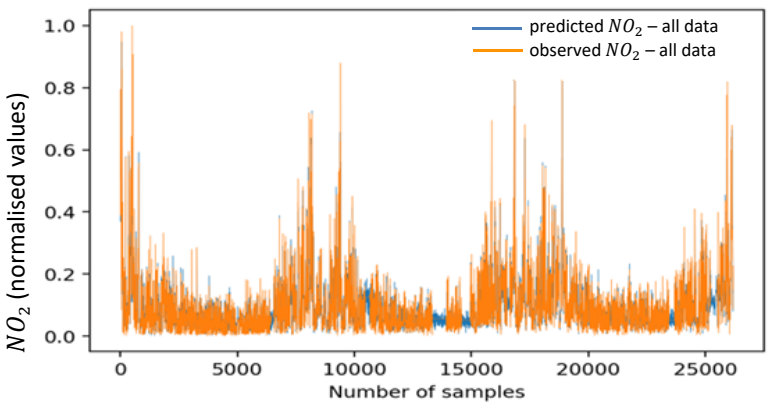

Sample

Figure 9. Relation between observed and predicted values $\mathrm{NO}_{2}$ for all data



$\mathrm{O}_{3}$ predicted values (normalised) - all data

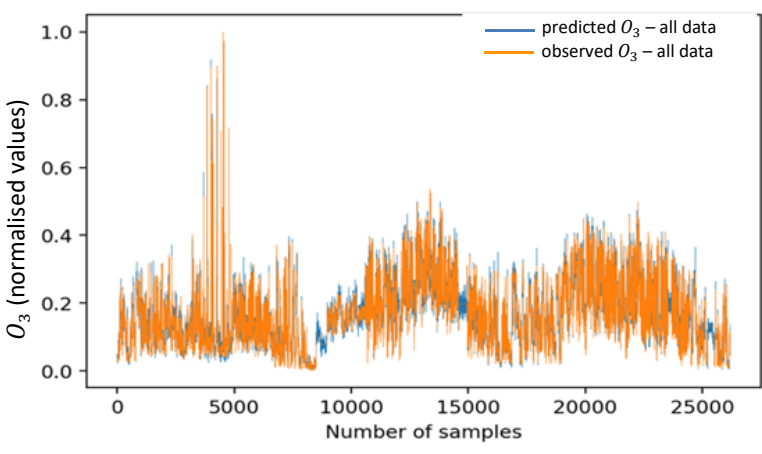

Sample

Figure 10. Relation between observed and predicted values $\mathrm{O}_{3}$ for all data
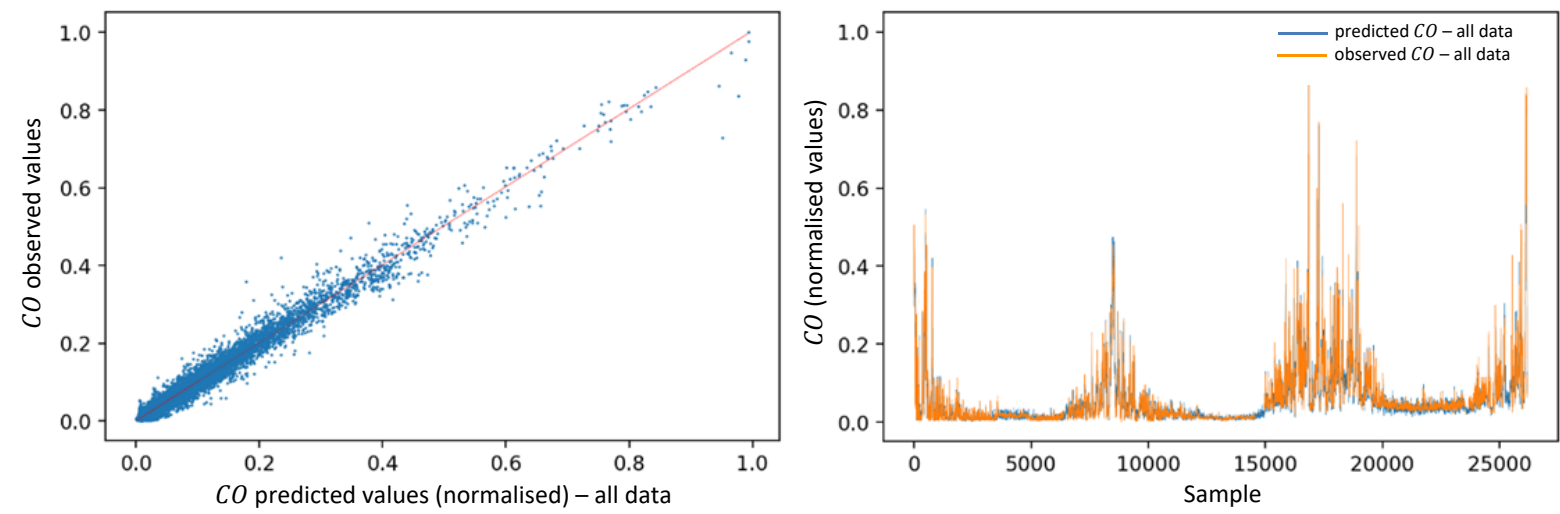

Figure 11. Relation between observed and predicted values $\mathrm{CO}$ for all data

It can be seen from Figures 7 to 11 that predicted values are very close to observed values. The observed values of the air pollutant concentration show seasonal changes that are repeated in each of the three years. The predicted values follow the seasonality of the observed values, so it can be said that optimal model 3 is suitable for prediction of the

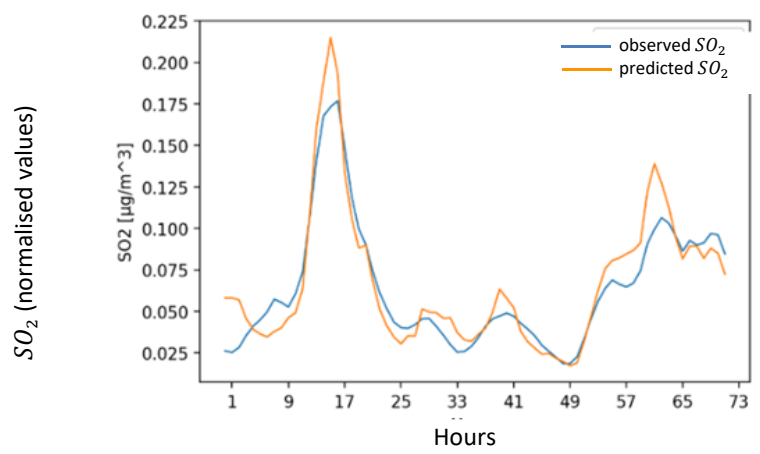

a) concentration of air pollutants for the 72 nd hour ahead.

In order to reach better visibility and for a randomly selected 72 consecutive hours the relation between predicted and observed values of concentration of all 5 air pollutants individually is graphically presented in Figure 12.



b) 


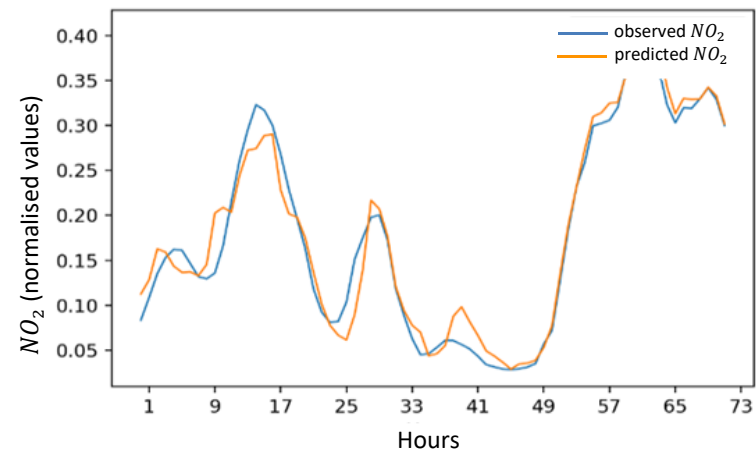

C)

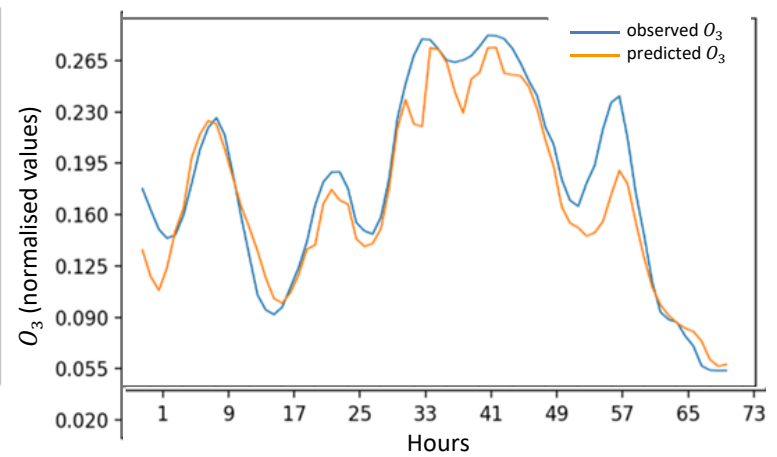

d)

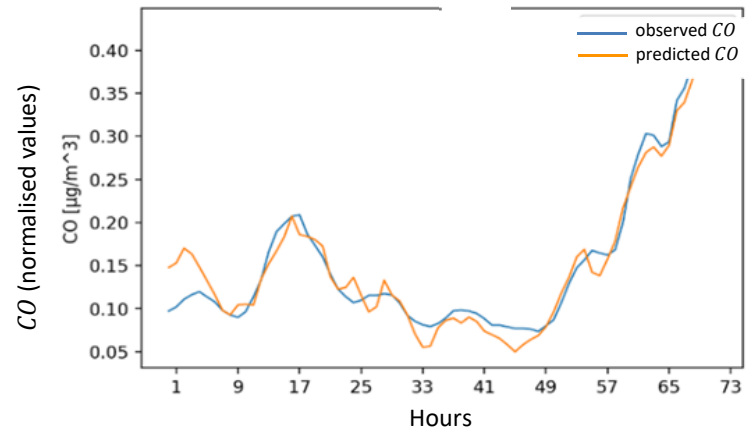

e)

Figure 12. Relation between observed and predicted values of air pollutants concentrations for randomly selected 72 consecutive hours (model 3): a) $\mathrm{SO}_{2}$, b) $\mathrm{PM}_{10}$, c) $\mathrm{NO}_{2}$, d) $\mathrm{O}_{3}$, e) $\mathrm{CO}$

Prediction of concentration of air pollutants for the 72nd hour ahead is a complex task, since it is a further hour ahead. Optimal model 3 can be used to predict the concentration of all 5 air pollutants used in this paper for each hour in the next 72 hours relative to the current hour, with the correct selection of the referent hour. Model 3 for the prediction of air pollutant concentration for 72nd hour ahead shows high prediction accuracy.

\section{Conclusion}

The NARX-SP neural network has proven to be a powerful tool for air pollutant concentrations prediction. For the selected optimal model the value of $R^{2}$ is very high and the value of MSE is very low. It can be concluded that the optimal model can predict concentration of air pollutants for 72nd hour ahead with a very high accuracy. The graphical presentations of the relations between observed and predicted values show that the predicted values deviate very little from the observed values confirming the conclusion that the optimal model prediction is very accurate.
As predicted values follow the seasonal changes of observed values, it can be concluded that NARX-SP neural network recognizes changes in observed values and creates functional dependence of the air pollutants concentration, meteorological parameters, month in the year, day of the week and time of the day. Optimal neural network model can predict with great accuracy sudden changes in the concentration of air pollutants. Therefore, it can be concluded that the use of a NARX-SP neural network can establish functional dependencies, complex and nonlinear relationship between inputs and outputs that do not have a mathematically defined functional dependency. 


\section{References:}

[1]. United States Environmental Protection Agency. (n.d.). Initial List of Hazardous Air Pollutants with Modifications. Retrieved from: https://www.epa.gov/haps/initial-list-hazardous-airpollutants-modifications [accessed: 20 February 2020].

[2]. Adams, M. D., \& Kanaroglou, P. S. (2016). Mapping real-time air pollution health risk for environmental management: Combining mobile and stationary air pollution monitoring with neural network models. Journal of environmental management, 168, 133-141.

[3]. Alimissis, A., Philippopoulos, K., Tzanis, C. G., \& Deligiorgi, D. (2018). Spatial estimation of urban air pollution with the use of artificial neural network models. Atmospheric Environment, 191, 205-213.

[4]. Zhang, B., \& Li, W. (2019). Intelligent air quality detection based on genetic algorithm and neural network: An urban China case study. Concurrency and Computation: Practice and Experience, 31(10), e4673.

[5]. Kolehmainen, M., Martikainen, H., \& Ruuskanen, J. (2001). Neural networks and periodic components used in air quality forecasting. Atmospheric Environment, 35(5), 815-825.

[6]. Cai, M., Yin, Y., \& Xie, M. (2009). Prediction of hourly air pollutant concentrations near urban arterials using artificial neural network approach. Transportation Research Part D: Transport and Environment, 14(1), 32-41.

[7]. Viotti, P., Liuti, G., \& Di Genova, P. (2002). Atmospheric urban pollution: applications of an artificial neural network (ANN) to the city of Perugia. Ecological Modelling, 148(1), 27-46.
[8]. AlHamaydeh, M., Choudhary, I., \& Assaleh, K. (2013). Virtual testing of buckling-restrained braces via nonlinear autoregressive exogenous neural networks. Journal of computing in civil engineering, 27(6), 755-768.

[9]. Marsland, S. (2015). Machine learning: an algorithmic perspective. CRC press.

[10]. Skansi, S. (2018). Introduction to Deep Learning: from logical calculus to artificial intelligence. Springer.

[11]. Mahmoud, S., Lotfi, A., \& Langensiepen, C. (2013). Behavioural pattern identification and prediction in intelligent environments. Applied Soft Computing, 13(4), 1813-1822.

[12]. Kumpati, S. N., \& Kannan, P. (1990). Identification and control of dynamical systems using neural networks. IEEE Transactions on neural networks, 1(1), 4-27.

[13]. Karasu, S., Altan, A., Saraç, Z., \& Hacıoğlu, R. (2017). Estimation of fast varied wind speed based on NARX neural network by using curve fitting. International Journal of Energy Applications and Technologies, 4(3), 137-146.

[14]. Lin, T. N., Giles, C. L., Horne, B. G., \& Kung, S. Y. (1997). A delay damage model selection algorithm for NARX neural networks. IEEE Transactions on Signal Processing, 45(11), 2719-2730.

[15]. Siegelmann, H. T., Horne, B. G., \& Giles, C. L. (1997). Computational capabilities of recurrent NARX neural networks. IEEE Transactions on Systems, Man, and Cybernetics, Part B (Cybernetics), 27(2), 208-215.

[16]. European Environment Agency. (2019, October 16). Air quality in Europe - 2019 report. Retrieved from: https://www.eea.europa.eu/publications/airquality-in-europe-2019 [accessed: 10 March 2020]. 